\title{
INSOLVENZ
}

\section{Scharfes Instrument}

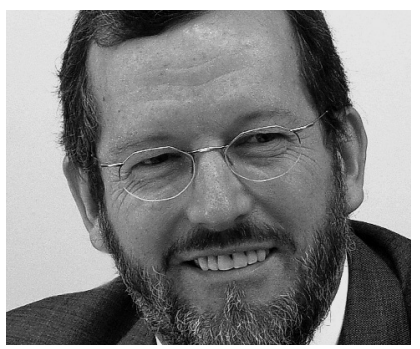

VON MARTIN BECK Prof. Martin Beck, Großhandelskaufmann und Diplombetriebswirt (FH), war Geschäftsführer im Diakonischen Werk Württemberg, Gründer und Geschäftsführer der Beratungsgesellschaft BSU und Sanierer in der Industrie. Er ist jetzt tätig als selbstständiger Unternehmensberater, Autor, Aufsichtsrat und Hochschullehrer. Internet http://www.prof-beck.net

Insolvenz bezeichnet die Situation eines Unternehmens, wenn es seine Zahlungsverpflichtungen gegenüber Mitarbeiterschaft, Kunden und Lieferanten nicht mehr erfüllen kann. Die Insolvenz ist gekennzeichnet durch akute Zahlungsunfähigkeit, drohende Zahlungsunfähigkeit und Überschuldung. Die Sozialwirtschaft blieb bislang weitgehend verschont von Insolvenzen, doch das Blatt scheint sich zu wenden.

Das Thema Insolvenz löst beim Publikum Ängste oder Erwartungen aus: Lästige Verpflichtungen abwerfen und unangenehme Gläubiger los werden, aber auch Gefahr für Arbeitsplätze oder für den Fortbestand von Diensten und Einrichtungen.

Da ist was dran. Mit der Insolvenz sollte man nicht spielen. Wenn erst einmal das Gericht und dann der vorläufige Verwalter und schließlich die Gläubiger das Sagen haben, dann sind die seitherigen Eigentümer aus dem Spiel und das seitherige Management ist entmachtet und nicht selten auch des Hauses verwiesen. Zurück bleiben Mitarbeiter, Kunden, Klienten und Lieferanten - sozusagen heimatlos und fremden Mächten ausgeliefert.

Die Sozialwirtschaft hat bisher wenig Bekanntschaft mit dem scharfen Instrumentarium der Insolvenz gemacht. Es sind weithin private Kleinstunternehmen, die scheitern, nicht die mittleren und großen Sozialwerke, von wenigen Ausnahmen abgesehen. Das wird aber so nicht bleiben (siehe auch der Beitrag von Wolfgang Lange in SOZIALwirtschaft aktuell 12/2010 zu Insolvenzen). Weil im allgemeinen Branchengefühl bisher nicht so viel passiert ist (obwohl in den vergangen Jahren viele Dutzend Sozial-Insolvenzen zu verzeichnen waren), kursieren immer wieder naive Insolvenzwunder-Geschichten. „Dann machen wir halt eine Insolvenz und sind alle Schulden los! « kann man da hören. Das stimmt zwar, aber man hat dann nicht nur die Schulden los, sondern in der Regel auch das Unternehmen.

Die Kirchen haben lange durch Geld verhindert, dass bei Caritas und Diakonie größere Insolvenzen eingetreten sind. Es herrschte eine Art panische Angst vor dem Insolvenzteufel, der, so glaubte man fest, für ein dramatisches Abnehmen der Kreditwürdigkeit sorgen würde, wenn man ihm eine Chance gäbe. Das war für die Beteiligten angenehm, aber es war pädagogisch falsch, denn dadurch wurden auch Managementfehler und Aufsichtsversagen zugedeckt. Jetzt fehlt das Geld für diese milden Taten - und das ist gut so. Ein fußkrankes Unternehmen wird nicht durch Vitamingaben gesund. Manchmal ist hartes Training nötig, und notfalls auch eine Amputation. Bei den weltlichen Wohlfahrtsverbänden gab es diese Goldesel nicht. Sie haben deshalb auch mehr Insolvenzen zu verzeichnen. Das ist bitter, aber manchmal reinigend.

Die Finanzlage der kommunalen Kassen wird das Insolvenzrisiko steigen lassen. Wenn für sicher gehaltene Finanzmittel ausbleiben, strauchelt manches Sozialunternehmen schnell. Dabei sind kleinere Unternehmen mehr gefährdet als große und Einproduktunternehmen mehr als solche, die fachlich diversifi- 


\section{Risiken für Aufsichtsgremien im Zusammenhang einer möglichen Insolvenz}

- Insolvenzverschleppung ist ein Straftatbestand, kein Kavaliersdelikt.

- Verdrängen oder ignorieren von Risiken kann existenzgefährdend sein.

- Gutmütig sein, statt strenge Fragen zu stellen, ist die falsche Art der Aufsicht.

- Die »vielen Zahlen« lästig finden, das kann man sich heute nicht mehr leisten.

- Wer sich überfordert und überlastet fühlt und deshalb die Aufgabe verweigert, läuft vor der Gefahr weg.

Was im Vorfeld getan werden kann, um eine Insolvenzgefahr zu erkennen und die Insolvenz zu vermeiden

- Ratschläge für Aufsichtsgremien

- Nicht alles glauben, was Ihnen vom Vorstand präsentiert wird

- Im Zweifel auch externen Sachverstand auf Kosten des Unternehmens einholen, wenn Sie dem Vorstand nicht (mehr) trauen oder ihn für überfordert halten
- Nicht wegschauen, sondern hinschauen!

- Ihre Aufgabe ist es nicht, gutmütig und lieb zu sein, sondern Unheil zu verhindern!

- Lieber der Führung lästig werden, als etwas versäumen!

- Fragen, fragen, fragen!

Ratschläge für hauptamtliche Vorstände und Geschäftsführer

- Lieber zu viel und zu früh informieren, als zu wenig und zu spät!

- Notfalls dem Aufsichtsgremium auch lästig werden, wenn dort keine große Lust auf Problem herrscht

- Nicht wegschauen, sondern hinschauen!

- Im Zweifel externen Sachverstand hinzuziehen, wenn Sie sich Ihres Risikopotenzials nicht sicher sind

- Ihre persönlichen Risiken frühzeitig prüfen und notfalls eigenmächtig tätig werden (als $\mathrm{GmbH}$-Geschäftsführung sind Sie ohnehin alleine für die rechtzeitige Insolvenzanmeldung verantwortlich)

\section{Wer muss die Insolvenz anmelden?}

\begin{tabular}{|l|l|l|l|}
\hline & GmbH & Verein & Stiftung \\
\hline Mitgliederversammlung & Nicht vorhanden & Keine Anmeldeplicht & Keine Anmeldepflicht \\
\hline Gesellschafterversammlung & Keine Anmeldepflicht & Nicht vorhanden & Nicht vorhanden \\
\hline Aufsichtsgremium & Nicht vorhanden & $\begin{array}{l}\text { Falls vorhanden: keine An- } \\
\text { meldepflicht }\end{array}$ & Nicht vorhanden \\
\hline Geschäftsführung & Anmeldepflicht & $\begin{array}{l}\text { Falls vorhanden: } \\
\text { keine Anmeldepflicht }\end{array}$ & $\begin{array}{l}\text { Falls vorhanden: } \\
\text { keine Anmeldepflicht }\end{array}$ \\
\hline Vorstand & Nicht vorhandenVorstand & Anmeldepflicht & Anmeldepflicht \\
\hline
\end{tabular}

\section{Mögliche Vorteile einer Insolvenz}

- Entlastung von drückenden Schulden

- Befreiung von hemmenden Arbeitsrechtsregeln

- Leichter Abwurf von betrieblichem Ballast

- Leichte Bereinigung von personellen Altlasten

Aber: Sie sind nicht mehr Herr im Hause! Sie sind ganz von Dritten abhängig. Und Sie werden sehr wahrscheinlich als Führung oder Aufsichtsgremium sofort vom Insolvenzverwalter von Ihren Aufgaben entbunden, Sie erhalten nur noch ein Gehalt nach der Versicherungshöchstgrenze und im schlimmsten Fall haben Sie auch Hausverbot und finden sich als Beschuldigter in einem Insolvenzverfahren wieder.

\section{Nachteile einer Insolvenz}

- Die Insolvenz dient in der Regeln den Gläubigern und soll ihr Vermögen ganz oder teilweise schützen.

- Sie sind ganz in der Hand des (vorläufigen oder endgültigen) Insolvenzverwalters.

- Der Insolvenzverwalter ist freier als jeder Unternehmer, aber er muss die Interessen der Gläubiger befriedigen. Kann er das nach deren Meinung nicht oder passt den Gläubigern seine Vorgehensweise nicht, kann er abgewählt werden.
- Alle Eigentümerrechte ruhen mit der Anmeldung der Insolvenz. Sie haben nichts mehr zu sagen, solange das Verfahren läuft - und hinterher vermutlich auch nicht.

- Alle Arbeitsverhältnisse sind ab sofort auf dem Prüfstand. Der vorläufige Verwalter wird die 30 Tage, in denen Insolvenzgeld von der Bundesagentur gezahlt wird, nutzen, um alles zu bereinigen, was er für überflüssig hält.

- Die Öffentlichkeitswirkung ist immer negativ. Mit etwas Glück und einem tüchtigen, fortführungswilligen Verwalter kann sich das wieder drehen.

- Alle internen und externen Beteiligten sind zunächst hoch verunsichert (Mitarbeiter, Klienten und deren Angehörige, Lieferanten, öffentliche und private Partner, Spender etc.).

\section{Gefährdungspotenziale in der Sozialwirtschaft}

Gefährdet sind grundsätzlich:

- alle »kirchlichen « Sozialwerke, die von ihren Verbänden oder Kirchen zur Beachtung von nicht refinanzierten Tarifwerken gezwungen werden

- alle "freien « Sozialwerke, die zwar theoretisch flexible Personalkosten haben, aber nicht streng auf den Zusammenhang von eingehendem und ausgehendem Geld achten (Controlling) 
ziert arbeiten. Für Vorstände und Geschäftsführungen gilt: Risikomanagement betreiben! Lieber zu früh als zu spät handeln! Alte Zöpfe abschneiden! Für Aufsichtsgremien gilt: Nicht alles glauben, was präsentiert wird! Fragen, fragen, fragen!

Ansonsten bleibt die Feststellung, dass Sozialunternehmen nicht für das ewige Leben geschaffen sind. Sie haben ihre Zeit, wie alle vom Menschen geschaffenen Organisationen. Die Frage ist nur, ob sie das selber merken und rechtzeitig aussteigen oder ob erst das Dach über ihnen einstürzen muss. an den Markt zurück. Weil das so ist, gibt es vielerorts, auch in der Sozialwirtschaft, große Hoffnungen auf die Insolvenz als Allheilmittel - eine unkritische Heilserwartung, wie nachfolgende Prüfung zeigt.

\section{Was bei einer Insolvenz passiert}

Die rechtliche Lage ist eindeutig: Es gibt Insolvenzgründe und es gibt Handlungsfristen. Da gibt es wenig zu diskutieren. Anders als früher im guten alten Konkursrecht hat die gefährdete Firma aber auch Wahlrechte, nicht nur Not-

\section{"Für Vorstände und Geschäftsführungen gilt: Lieber zu früh als zu spät handeln!«}

In den alten Zeiten, als es noch königliche Kaufleute gab und Ehrbarkeit und Verlässlichkeit keine Charakterschwächen, sondern Qualitätsmerkmale von Unternehmern waren, war der »Bankrott« nicht nur das Todesurteil für die Firma, sondern auch für die Reputation der Unternehmerfamilie - und nicht selten schritt der Patron nach der Anmeldung der Zahlungsunfähigkeit gemessenen Schrittes in den nahen Stadtpark, um sich dort mit der mitgebrachten Pistole von der Schande des Bankrotts zu befreien. In Thomas Manns Kaufmannsroman »Die Buddenbrooks « wird aus dieser vergangenen Welt saftig und farbig berichtet.

Der Gesetzgeber hat seither viel getan, um die Insolvenz, wie die prekäre Situation heute gesetzlich genannt wird, flexibler, sachgerechter und auch produktiv nutzbarer zu machen. Nicht wenige Insolvenzanwälte verstehen sich in erster Linie als » Fortführer «, während noch vor einer Generation das Zerlegen und Zerschlagen von Restvermögen und Firma an der Tagesordnung war. Gute Verwalter führen die von ihnen im Auftrag des Insolvenzgerichts und der Gläubiger geleiteten Unternehmen oft jahrelang, knien sich in die jeweilige Branche hinein und geben dann nach getaner Arbeit ein verschlanktes, fit gemachtes und wieder überlebensfähiges Unternehmen fallpflichten. Sie darf zum Beispiel schon bei drohender Zahlungsunfähigkeit anmelden, sozusagen strategisch, im Vorfeld. Und sie kann eine Planinsolvenz anstreben, bei der mit der Insolvenzanmeldung gleich ein Lösungsvorschlag mitgeliefert wird. Die Wahrheit ist allerdings, dass bisher in Deutschland nur eine kleine Zahl von Planinsolvenzen angenommen und erfolgreich durchgeführt wurden. Die Hürden sind hoch und die Chancen begrenzt.

\section{Ein Dummer muss gefunden werden}

Umgangssprachlich lässt es sich so beschreiben: Ein Unternehmen, das durch eigene oder fremde Schuld zahlungsunfähig oder überschuldet wurde, unterstellt sich dem Schutz des Insolvenzrechts und dem Kommando eines allmächtigen Insolvenzverwalters, beides mit dem Ziel, Schulden und andere Lasten abzuwerfen, Kosten zu senken und die unternehmerische Freiheit wieder zu gewinnen.

Dabei gehen allerdings meist die Gesellschafter und häufig die Führungspersonen verloren. Das Ganze funktioniert auch nur, wenn einer (populär gesagt: »ein Dummer «) gefunden wird, der durch Reduzierung oder Vernichtung seiner eigentlich berechtigten Forderun- gen geschädigt wird, damit die insolvente Firma danach erleichtert wieder weitermachen kann. Es geht also um eine Art unfreiwilliger Umverteilung, bei der die meldepflichtigen Personen eine große Verantwortung für Vermögen und Existenz Dritter tragen.

\section{Wie viele Insolvenzen es bisher in der Sozialwirtschaft gibt}

Der Autor hat vor dieser Veröffentlichung versucht, von einigen Spitzenführungskräfte der Sozialwirtschaft Antworten auf folgende Fragen zu erhalten:

Erstens: Gab es in Ihrem Verantwortungsbereich in den vergangenen fünf Jahren eine Insolvenz?

Zweitens: Gab es Versuche, Insolvenzen abzuwehren? Erfolgreich?

Drittens: Führten die Insolvenzen zu einem Neustart oder war das (soziale) Unternehmen anschließend verloren?

Viertens: Wenn es eine zweite Chance gäbe, wie würde man sich heute auf diesen Fall vorbereiten?

Zwei Kollegen haben besonders schnell geantwortet:

- Peter Schimpf, Landesgeschäftsführer, Parität Bayern, München: Es wird kein Fall aus dem Bereich des Paritätischen in Bayern genannt, wohl aber ein selbst erlebter Fall in der Diakonie von Bremen, der nicht abgewendet werden, aber durch eine Nachfolgegesellschaft teilweise abgefedert werden konnte.

- Weihbischof Dr. Bernd Uhl, Vorsitzender des Caritasverbandes für die Erzdiözese Freiburg: »Im Bereich des Caritasverbandes für die Erzdiözese Freiburg, dessen erster Vorsitzender ich bin, gab es meiner Erinnerung nach in den letzten fünf Jahren keinen Insolvenzfall. « (Außerhalb der Diözese wird noch das Müttererholungsheim in Bad Griesbach genannt, das vor zwei Jahren in Insolvenz ging).

Während der Vorbereitung auf diese Veröffentlichung rief der Autor zudem in seinem E-Mail-Newsletter, der überwiegend von Führungskräften der Wohlfahrt gelesen wird, zur Nennung von Insolvenzen aus der jüngeren Vergangenheit der Sozialwirtschaft auf. Innerhalb von wenigen Wochen kamen auf diese Weise mehr als 50 Fälle von In- 
solvenzen in der gemeinnützigen Sozialwirtschaft zusammen. Die weit überwiegende Mehrheit dieser Fälle stammt aus dem Umfeld von Deutschem Roten Kreuz und Arbeiterwohlfahrt. Diakonie und Caritas haben bisher (wie bereits erwähnt) aus einer Art Reflexfurcht vor den Banken, fast alle Insolvenzen vermieden, teilweise um den Preis hoher finanzieller Einsätze. Die Recherchen laufen weiter. In absehbarer Zeit wird es wohl möglich sein, einen ersten Überblick über die Insolvenzgeschichte der Sozialwirtschaft zu präsentieren und daraus weitere belastbare Ratschläge für Verantwortliche sozialer Unternehmen zu ziehen.

\section{Was auf uns zukommt - drei Vorhersagen}

Der Autor wagt den folgenden, unwissenschaftlichen, aber erfahrungsgesättigten Versuch einer prophetischen Vorausschau auf die nächsten Insolvenz-Jahre in der Sozialwirtschaft:

Erstens: Die nicht kirchlich gebundenen Wohlfahrtsverbände haben schon viele »kranke « Mitglieder abgeworfen oder durch Insolvenz verloren. Heute stehen sie schlanker und "gereinigt « da.

Zweitens: Die kirchlichen Wohlfahrtsverbände haben diesen schmerzhaften Reinigungsprozess noch vor sich. Die Bereitschaft und die Kraft der Kirchen, vermeintlich peinliche Vorgänge durch Geld oder durch sanften Druck in Richtung Übernahme zu vermeiden, wird weiter abnehmen.

Drittens: Das höchste Risiko haben kleine und mittelgroße Sozialunternehmen in Ländern mit strenger Kommunalisierung. Sie sind den örtlichen Machtverhältnissen und der Kassenlage der Landkreise und Städte weitgehend schutzlos ausgeliefert.

\section{Gutes Geld für gute Zwecke.} Moderne Konzepte für soziales Wagniskapital.

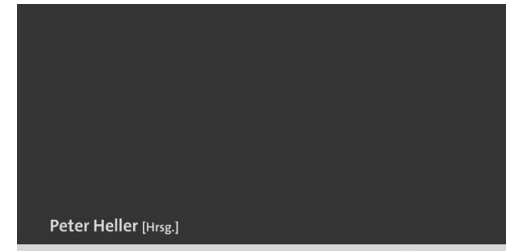

Innovative Funding

Mechanisms for

Social Change

$$
\text { Nomos }
$$

\author{
Innovative Funding \\ Mechanisms for Social \\ Change \\ Herausgegeben von \\ Dr. Peter W. Heller \\ 2009, 144 S., brosch., 24,-€ \\ ISBN 978-3-8329-3948-9
}

Wo die soziale Sicherung des Staates versagt, findet die Zivilgesellschaft ihre eigenen Lösungen. In vielen Ländern haben zivilgesellschaftliche Organisationen mehr Arbeitsplätze geschaffen als der öffentliche und der private Sektor. Um den damit verbundenen Herausforderungen gerecht zu werden, orientieren sich soziale Pioniere an den erfolgreichen Geschäftsmodellen und Management-Strategien der privaten Unternehmen, sie werden zu „social entrepreneurs“. Aber oft fehlen ihnen die finanziellen Mittel, um ihr soziales Unternehmen auf einen stabilen Wachstumskurs zu bringen.

Dieses Buch untersucht das Potential maßgeschneiderter sozialer Investmentfonds und Investitionsstrategien von Stiftungen, um auf neuen Wegen das fehlende Kapital bereitzustellen. Die Themen der portraitierten Finanzierungsmodelle reichen von der Augenheilkunde über die biologische Landwirtschaft bis zur Pressefreiheit. Die Autoren untersuchen die Voraussetzungen ihrer Entwicklung und ihre Relevanz für die Zukunft der Zivilgesellschaft.

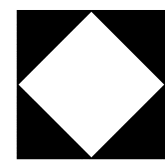

